\title{
A Pair of Oviduct-Born Pickpocket Neurons Important for Egg-Laying in Drosophila melanogaster
}

\author{
Hyunjin Lee ${ }^{1,2}$, Hyun Woo Choi ${ }^{1,2}$, Chen Zhang ${ }^{1}$, Zee-Yong Park ${ }^{1, *}$, and Young-Joon Kim ${ }^{1, *}$
}

\begin{abstract}
During copulation, male Drosophila transfers Sex Peptide (SP) to females where it acts on internal sensory neurons expressing pickpocket (ppk). These neurons induce a post-mating response (PMR) that includes elevated egglaying and refractoriness to re-mating. Exactly how ppk neurons regulate the different aspects of the PMR, however, remains unclear. Here, we identify a small subset of the ppk neurons which requires expression of a pre-mRNA splicing factor CG3542 for egg-laying, but not refractoriness to mating. We identify two CG3542-ppk expressing neurons that innervate the upper oviduct and appear to be responsible for normal egg-laying. Our results suggest specific subsets of the ppk neurons are responsible for each PMR component.
\end{abstract}

\section{INTRODUCTION}

Egg-laying is a key reproductive behavior in many invertebrates that depends on the coordinated action of multiple physiological and behavioral steps associated with egg formation, ovulation, and oviposition. Like reproduction in mammals, each step of egg-laying is influenced by endocrine factors; juvenile hormone $(\mathrm{JH})$ is required for egg formation (Bownes, 1989; Gruntenko et al., 2010; Sliter et al., 1987), and octopamine is required for ovulation (Monastirioti, 1996; 2003; Rodri et al., 2006). Even neurons producing Insulin-like peptide 7 (Ilp7) have been linked to oviposition in Drosophila melanogaster (Yang et al., 2008).

As with many other animals, egg-laying in Drosophila is tightly associated with mating. After a female mates and receives sperm from a male, she becomes refractory to further mating and begins to lay eggs. This behavioral switch, which is known as the post-mating response (PMR), is induced by Sex Peptide $(\mathrm{SP})$ in the seminal fluid transferred to females during insemination (Aigaki and Fleischmann, 1991; Chen et al., 1988; Liu and Kubli, 2003). Females that mate with males lacking SP lay few

${ }^{1}$ School of Life Sciences, Gwangju Institute of Science and Technology, Gwangju 61005 , Korea, ${ }^{2}$ These authors contributed equally to this work.

*Correspondence: kimyj@gist.ac.kr (YJK); zeeyong@gist.ac.kr (ZYP)

Received 10 May, 2016; revised 25 May, 2016; accepted 25 May, 2016; published online 24 June, 2016

Keywords: CG3542, egg-laying, phosphoprotein, pickpocket, post mating response, sex peptide eggs and mate multiple times (Peng et al., 2005a). SP is also associated with other behavioral and physiological changes, such as feeding, sleep and immune responses (Carvalho et al., 2006; Domanitskaya et al., 2007; Isaac et al., 2010; Peng et al., 2005b; Ribeiro and Dickson, 2010; Walker et al., 2015).

SP acts via a G-protein coupled receptor called SP receptor (SPR) (Yapici et al., 2008). SPR expression in pickpocket (ppk) neurons is required for a normal PMR, and the activation of SPR in ppk neurons is sufficient to induce the PMR even in virgin flies. The female genital tract houses 30-38 ppk neurons. Silencing of these neurons induces a PMR similar to that caused by mating or SPR activation. This indicates activation of SPR suppresses the activity of the ppk neurons (Häsemeyer et al., 2009; Yang et al., 2009). ppk is co-expressed in female reproductive organs with fruitless (fru) and/or doublesex (dsx), two genes encoding transcription factors important for sex determination (Häsemeyer et al., 2009; Rezával et al., 2012; Yang et al., 2009). The ppk gene encodes an amiloride-sensitive sodium channel important for mechanosensation (Adams et al., 1998; Zhong et al., 2010). Because of this, peripheral neurons in the legs, wings, and genital tract that express ppk are generally considered sensory neurons that signal to the central brain. Still, even though SP and SPR in ppk neurons clearly direct the various components of the PMR, it is unclear which ppk neurons regulate the distinct behaviors of mating receptivity and egg-laying.

As a GPCR, SPR appears to be coupled with Gai or Gao, both of which reduce levels of the intracellular second messenger cAMP (Yapici et al., 2008). This drop in cAMP, in turn, modulates the activity of cAMP-dependent protein kinase $A$ (PKA). Thus, we reasoned SPR activation should result in global changes in the phosphorylation state of many proteins, including important mediators downstream of SPR. In this study, we took a mass spectrometry-based proteomic approach to identify phosphoproteins downstream of SPR signaling. After identifying proteins whose phosphorylation status changes in response to SP treatment, we examined the functions of these proteins using RNAi. We found that CG3542 (Mount and Salz, 2000; Zhu et al., 2005), which encodes a protein closely related to the premRNA processing factor PRP40, is important in ppk neurons for the increase in egg-laying of mated females but not for their reduced mating receptivity. Using an intersectional genetic approach, we identified a group of 2-3 ppk and CG3542 double-positive neurons that innervate the upper oviduct. Silencing of these neurons affects egg-laying but not mating receptivity in mated females. Our results suggest egg-laying and mating receptivity are regulated by distinct subsets of ppkneurons. 


\section{MATERIALS AND METHODS}

\section{Phosphoprotein analysis}

BG2 cells were obtained from Drosophila Genome Resource Center (Cell line stock number, 51), and cultured according to the protocol form the provider (https://dgrc.bio.indiana.edu/ include/file/general_maintenance.pdf). BG2 cells with or without SP treatment were lysed in protein extraction buffer containing $50 \mathrm{mM}$ Tris-HCl pH 8.2, $8 \mathrm{M}$ Urea, $75 \mathrm{mM} \mathrm{NaCl}, 1 \mathrm{mM} \mathrm{NaF}, 1$ $\mathrm{mM} \beta$-glycerophosphate, $1 \mathrm{mM}$ sodium orthovanadate, $1 \mathrm{mM}$ sodium pyrophosphate and $1 \mathrm{mM}$ PMSF. After sonication, protein concentrations were determined using a BCA Protein Assay Kit (Thermo Scientific, UK). Protein samples (100 $\mu \mathrm{g}$ each) were chemically reduced (5 mM, DTT) and alkylated $(25 \mathrm{mM}$, IAA) prior to trypsin digestion. After 4-fold dilution with $25 \mathrm{mM}$ Tris- $\mathrm{HCl}$ buffer ( $\mathrm{pH} 8.2$ ), the protein samples were enzymatically digested using a sequencing grade trypsin at an enzyme to substrate ratio of $1: 50$ at $37^{\circ} \mathrm{C}$ for $16 \mathrm{~h}$. Digestion reaction was stopped by the addition of $90 \%$ formic acid to a final concentration of $1 \%$. Resulting tryptic digest peptide samples were desalted for subsequent phosphopeptide enrichment procedure. Phosphopeptides enriched with IMAC POROS 20 MC were subsequently subjected to the micro RPLC-MS/MS analysis described previously (Choi et al., 2011). Briefly, analyses of phosphopeptide-enriched samples were performed using Agilent 1100 Series high-performance liquid chromatography (HPLC) (Agilent Technologies) coupled to a linear quadrupole ion trap mass spectrometer (LTQ, Thermo Finnigan, USA). The resulting MS/MS spectra searched against a composite protein database containing the IPI mouse protein database (v.3.28), and its reversed complement using SEQUEST algorithm (Sorcerer system). Differential modification search options for phosphorylation modification (+80 on Ser, Thr, Tyr), and oxidation (+16 on Met), were considered in the search. 'Peptide prophet' and 'Protein prophet' scores of $90 \%$ was used to filter the search results. We applied the log2(protein ratio) from relative spectral counts (Rsc) for label free quantification (Old et al., 2005). $R S C=\log 2[(n 2+f) /(n 1+f)]+\log 2[(t 1-n 1+f) /(t 2-n 2+$ $\mathrm{f})$ ] where, for each protein, RSC is the log2 ratio of abundance between Samples 1 and 2; $\mathrm{n} 1$ and $\mathrm{n} 2$ are spectral counts for the protein in Samples 1 and 2, respectively; t 1 and $t 2$ are total numbers of spectra over all proteins in control and SP-treated samples; and $f$ is a correction factor, set to 0.5 (Beissbarth et al., 2004). Ammonium bicarbonate, calcium chloride, dithiothreitol (DTT), iodoacetamide (IAA), sodium chloride, sodium orthovanadate $\left(\mathrm{Na}_{3} \mathrm{VO}_{4}\right)$, sodium fluoride $(\mathrm{NaF})$, phenylmethylsulfonyl fluoride (PMSF), sodium $\beta$-glycerophosphate, urea and ammonium dihydrogen phosphate $\left(\mathrm{NH}_{4} \mathrm{H}_{2} \mathrm{PO}_{4}\right)$ were purchased from Sigma (USA). Formic acid (FA) and trifluoroacetic acid (TFA) were obtained from Merck (Germany). HPLC grade acetonitrile (ACN), methanol and deionized water were all purchased from Fisher Science. Sequencing grade modified trypsin was obtained from Promega (USA). Aqua C18 (particle size $5 \mu \mathrm{m})$ reversed-phase column material was purchased from Phenomenx (USA). The IMAC POROS 20 MC was purchased from Applied Biosystems (USA).

\section{Fly strains}

We obtained the following RNAi transgenic lines from the Vienna Drosophila RNAi Center (VDRC) (Austria): CG14939-IR1 (VDRC stock number 107010), CG6983-IR1 (35159), CG5643IR1 (27470), CG3333-IR1 (34597), CG8594-IR1 (4642), CG8201-IR1 (52553), CG11621-IR1 (16240), CG3542-IR1 (26227), CG3542-IR2 (26229), CG10480-IR1 (38389),
CG2173-IR1 (36516), CG8108-IR1 (35343), CG11417-IR1 (18087), CG4236-IR1 (26455), CG6831-IR1 (40399), CG33979IR1 (21995), CG31678-IR1 (103705), CG6338-IR1 (12633), CG10033-IR1 (38320), CG42250-IR1 (33799), CG3696-IR1 (10762), CG6418-IR1 (40379), CG9191-IR1 (52549), CG30122IR1 (106984), CG16973-IR1 (101517), CG2691-IR1 (110718), CG6501-IR1 (105605), CG14616-IR1 (106973), CG8092IR1 (28196), and CG42783-IR1 (105624). Y. Jan (UCSF) provided ppk-Gal4. We combined ppk-GAL4 with UASDicer2 and used it as a ppk-specific driver for all the RNAi experiments. B.J. Dickson kindly provided UAS>stop $>n S y b-$ GFP, UAS >stop>DSCAM17.1-GFP and UAS>stop>Kir2.1. We also used the published lines UAS $>$ stop $>m C D 8-G F P(Y u$ et al., 2010); LexAop-FLP (Bussell et al., 2014) and UAS-mSP (Nakayama et al., 1997) in this study. To generate CG3542LexA and CG3542-GAL4, we performed a PCR amplification of the genomic region $5^{\prime}$ of the CG3542 transcriptional start site (+228 to -22) (forward primer, 5' NNGCGGCCGCCGATAA CAGAAAATCTACCGGGT-3'; reverse primer, 5'-NNTCTAGA CCCACGCTAGGAGGAACATT-3') and cloned the product into the $p E N T R$ vector (Invitrogen). We then recombined this into the pBPnlsLexAp65Uw vector for the LexA line or the pPTGAL4(+) vector for the GAL4 line (Pfeiffer et al., 2010). We injected each final plasmid into embryos using standard techiniqes for insertion into a specific site on the second chromosome (VIE-72A) using phiC31-mediated recombination.

\section{Behavioral assays}

We raised all flies on normal fly food at $25^{\circ} \mathrm{C}$ under a $12 \mathrm{~h}: 12 \mathrm{~h}$ light: dark cycle (Park et al., 2014). For all behavioral assays, we collected flies immediately after eclosion and aged females 3-5 days in groups of $\sim 10$ and males 4 days individually. For the initial mating assays, we paired virgin females individually with naïve Canton $S$ (CS) males in $1 \mathrm{~cm}$ diameter chambers and counted the number of mating pairs for $1 \mathrm{~h}$. In case of egglaying, we transferred individual females who copulated within 1 $h$ to vials containing normal fly food. Then, we counted the eggs laid by each female over the course of $48 \mathrm{~h}$. For virgin egglaying, we transferred 5 aged virgin females to vials containing normal fly food and counted the total eggs laid over the course of $48 \mathrm{~h}$. For re-mating, we paired individual females who had mated within the past $48 \mathrm{~h}$ with naïve CS males and observed their mating for $1 \mathrm{~h}$. All behavioral assays were performed at RT $\left(25^{\circ} \mathrm{C}\right)$ and $60 \%$ humidity.

\section{Immunohistochemistry}

We dissected ovaries in phosphate buffered saline (PBS) $(\mathrm{pH}$ 7.4) and fixed them in 4\% paraformaldehyde in PBS for $30 \mathrm{~min}$ at room temperature. After washing them with PBST, we incubated them with a rabbit anti-GFP antibody (1:1000; Invitrogen, A11122) for $48 \mathrm{~h}$ at $4^{\circ} \mathrm{C}$. After washing, we then incubated the samples for $24 \mathrm{~h}$ at $4^{\circ} \mathrm{C}$ with an Alexa 488-conjugated goat anti-rabbit (1:1000; Invitrogen, A11008) secondary antibody and an Alexa 555-conjugated phalloidin (1:1000; Invitrogen, A34055) to visualize actin in the muscle-rich reproductive tract. Finally, we mounted the tissues in Vectashield and imaged them with an LSM 700/Axiovert 200M confocal microscope (Zeiss). All confocal images are maximal intensity Z-projections. We performed all image processing with ImageJ.

\section{Statistics}

We used GraphPad Prism 5 to compare experimental groups with multiple control groups using the ANOVA followed by Tukey's test for multiple comparisons. 


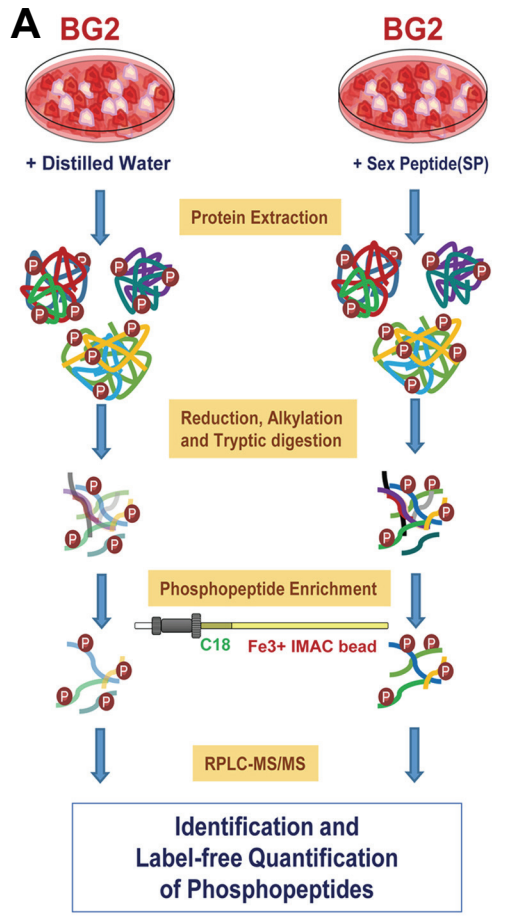

B

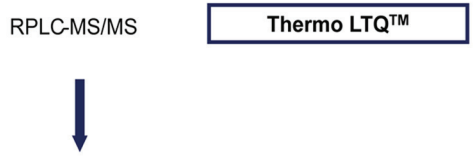

Identification

of phosphoproteins

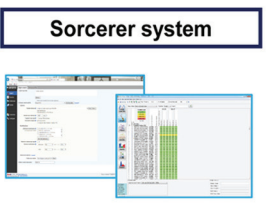

Label-free Quantification of phosphoproteins

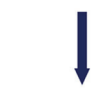

Differentially Expressed phosphoproteins

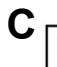

\begin{tabular}{|c|c|c|c|}
\hline $\begin{array}{l}\text { Protein } \\
\text { accession }\end{array}$ & Protein names & $\mathrm{R}_{\mathrm{SC}}{ }^{\mathrm{a}}$ & $p^{b}$ \\
\hline Q960C2 & SD07967p & -3.69443 & 0.00042 \\
\hline P276191 & Isoform 1 of Dynamin & -2.79955 & 0.004622 \\
\hline A1Z9X0 & Atypical protein kinase $C$, isoform $A$ & -2.79955 & 0.004622 \\
\hline Q9VR591 & $\begin{array}{l}\text { Isoforml of Inositohexakisphosphate } \\
\text { diphosphoinositol-pentakisphosphate } \\
\text { Kinase }\end{array}$ & -2.79955 & 0.004622 \\
\hline Q24572 & Probable histone-binding protein Caf1 & -2.31353 & 0.028992 \\
\hline Q9VPX6 & Adenylyl cyclase-associated protein & -2.31353 & 0.028992 \\
\hline Q7KVQ7 & $\begin{array}{l}\text { Transport and golgi organization } 5 \text {, } \\
\text { isoform B }\end{array}$ & -2.31353 & 0.028992 \\
\hline Q9VSK4 & CG6983 & -2.31353 & 0.028992 \\
\hline P32023-1 & $\begin{array}{l}\text { Isoform } \mathrm{CD} 5 \text { of } \mathrm{CGMP} \text { - dependent } \\
\text { protein kinase, isozyme } 2 \text { forms } \\
\mathrm{CD} 5 / \mathrm{T} 2\end{array}$ & -2.31353 & 0.028992 \\
\hline Q04688 & DNAbinding protein Ets97D & -2.31353 & 0.028992 \\
\hline Q9VXF9 & CG4420 & -2.31353 & 0.028992 \\
\hline Q7JNX6 & Phosphoinositide-3 kinase & -2.31353 & 0.028992 \\
\hline Q8IN05 & Epsinlike, isoform D & -2.31353 & 0.028992 \\
\hline Q9W1H5 & Decapping protein 1 & -2.31353 & 0.028992 \\
\hline A1ZAM0 & CG6665, isoform C & -2.31353 & 0.028992 \\
\hline Q24036 & Failed axon connections protein & -2.10887 & 0.046218 \\
\hline Q7JQN4 & Rs1 & -1.86726 & 0.054622 \\
\hline O44081-1 & $\begin{array}{l}\text { Isoform A of H/ACA ribonucleoprotein } \\
\text { complex subunit } 4\end{array}$ & -1.75952 & 0.056723 \\
\hline Q9VHCO & RNAbinding protein S1 & -1.7114 & 0.061345 \\
\hline Q8SX89 & Kugelkern, isoform A & -1.57955 & 0.07437 \\
\hline Q24156 & Transcription factor & -1.57716 & 0.07479 \\
\hline
\end{tabular}

D

\begin{tabular}{|c|c|c|c|}
\hline $\begin{array}{l}\text { Protein } \\
\text { accession }\end{array}$ & Protein names & $\mathrm{R}_{\mathrm{SC}^{\mathrm{a}}}$ & $p^{b}$ \\
\hline Q8MMD2 & Eps-15, isoform $B$ & 2.818757 & 0.002521 \\
\hline Q7JXU4 & Ngp & 2.818757 & 0.002521 \\
\hline Q7KU25 & CG3542, isoform B & 2.332728 & 0.020168 \\
\hline B7Z002 & Kismet, isoform C,CG3696 & 2.332728 & 0.020168 \\
\hline BTYZYO & CG31678, isoform C & 2.332728 & 0.020168 \\
\hline P46863 & Bipolar kinesin KRP130, CG9191 & 2.332728 & 0.020168 \\
\hline Q9VAF4 & Dim gammatubulin 1 & 2.332728 & 0.020168 \\
\hline O46307 & CG11417 & 2.332728 & 0.020168 \\
\hline Q9VB23 & Widerborst, isoform A, CG5643 & 2.332728 & 0.020168 \\
\hline Q8T4F7-4 & Isoform 4 of Protein enabled & 2.12809 & 0.045378 \\
\hline Q7JR96 & CG6410 & 1.59637 & 0.071849 \\
\hline A8JV09 & Pod1, isoform $F$ & 1.390527 & 0.10042 \\
\hline Q9VHY5 & $\begin{array}{l}\text { Transcription initiation factor TFIID } \\
\text { subunit } 7\end{array}$ & 1.390527 & 0.10042 \\
\hline P42207-1 & Isoform A of Septin 1 & 1.390527 & 0.10042 \\
\hline
\end{tabular}

${ }^{\text {a }} R_{\mathrm{SC}}$ calculated with $f 0.5$, comparing BG2_SP/BG2_DW $\log _{2}$ ratio.

${ }^{b} p$ value for $R_{\mathrm{SC}}$ (BG2_SP/BG2_DW).
Fig. 1. Identification of signaling molecules downstream of SPR via a mass spectrometry-based label-free phosphoproteomic approach. (A) Experimental scheme for phosphopeptide enrichment. (B) Experimental scheme for micro RPLC-MS/MS analysis and label-free quantification. (C) Proteins showing reduced levels of phosphorylation in SP-treated BG2 cells as filtered by $R_{\mathrm{SC}}$ significance $(p$ $<0.1$ ). (D) Proteins showing elevated levels of phosphorylation in SP-treated BG2 cells as filtered by $R_{S C}$ significance $(p<0.1)$.

\section{RESULTS}

In this study, we hoped to discover proteins downstream of SP and SPR actions as well as the neurons that express them. To apply our mass spectrometry-based phospho-proteomic approach, we needed a cell line that expresses SPR. After examining several cell lines derived from Drosophila melanogaster nervous tissue, we found that the BG2 cell line derived from third instar larval brains meets this criterion (data not shown). After treating BG2 cells with $100 \mathrm{nM} \mathrm{SP}$ for $2 \mathrm{~h}$, we analyzed them with label-free quantitative mass spectrometry. This technique permits the unbiased monitoring of protein phosphorylation levels at specific individual phosphorylation sites. We iden- tified 35 proteins that show significantly different levels of phosphorylation between SP- and vehicle-treated cells $(p<0.1)$. Consistent with our prediction that SPR activation would reduce PKA activity, we found more proteins with reduced phosphorylation (21) than increased phosphorylation (14) (Fig. 1).

To determine whether any of these proteins showing altered phosphorylation upon SPR activation affect the egg-laying or mating refractoriness components of the PMR in vivo, we performed a small-scale RNAi screen knocking-down each candidate in ppk neurons (Fig. 2A). In this screen, we found females expressing CG3542-IR1 show significantly elevated egg-laying for $48 \mathrm{~h}$ post-mating (Fig. 2B). Although knock-down of CG3542 affects egg-laying, it does not seem to affect receptiv- 
A

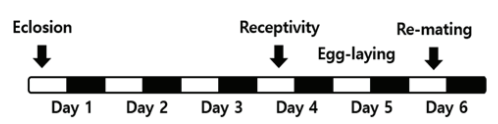

B

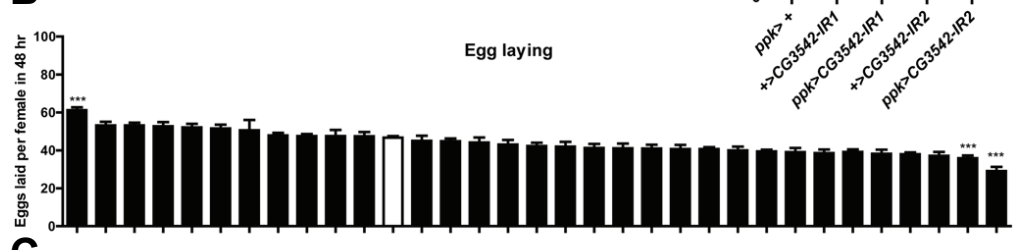

C

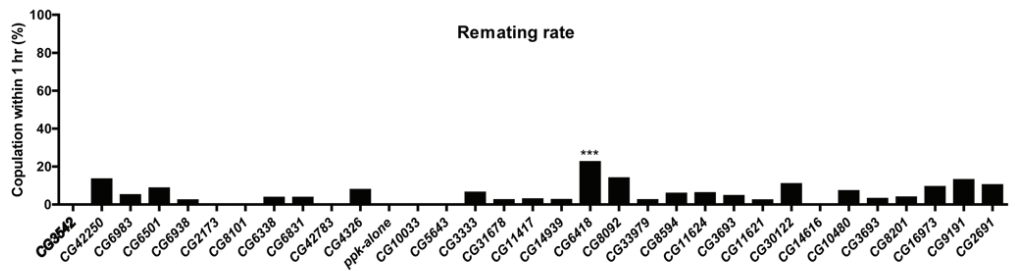

D

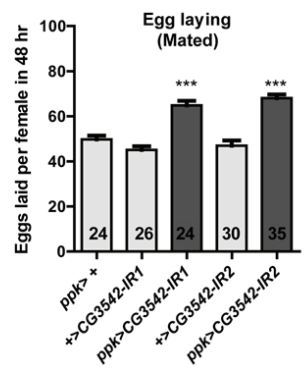

ppk>Gene-IR

Fig. 2. CG3542 knock-down in ppk neurons affects egg-laying in mated females. (A) Behavioral protocol used for $(B, C)$. (B) Eggs laid per female of the indicated UAS line crossed with ppk-Gal4 for 48 h post-mating, $n=20-58$ (n of CG8101$R N A i=12) .{ }^{* * *} p<0.001$ for comparisons against ppk-GAL4 alone (white bar); one-way ANOVA followed by Tukey's test for multiple comparisons. (C) Re-mating rate, $n=20-58$ (CG47283-RNA haven't be tested). ${ }^{* * *} p<0.001$ for comparisons against ppk-GAL4 alone; Fisher's exact test. (D) Eggs laid per female of the indicated genotype for $48 \mathrm{~h}$ post-mating, $n=20-35$. ${ }^{* * *} p<0.001$, oneway ANOVA followed by Tukey's test for multiple comparisons. ity to re-mating $48 \mathrm{~h}$ after initial mating (Fig. 2C). We confirmed the specificity of the RNAi-mediated knock-down of CG3542 by testing an additional RNAi line (CG3542-IR2) and observed a similar effect on egg-laying (Fig. 2D). This suggests CG3542 is important for the function of ppk neurons, especially the subset of the ppk neurons that direct post-mating changes in egglaying. CG3542 encodes a protein predicted to regulate mRNA splicing, but this has not yet been confirmed.

Since knock-down of CG3542 in ppk neurons affects egglaying but not re-mating, we reasoned that we should be able to affect egg-laying by manipulating the activity of ppk neurons positive for CG3542. To test this idea, we adopted an intersectional genetic approach. We generated a CG3542-LexA transgene (Fig. $3 \mathrm{~A}$ ) and combined it with LexAOP-FLP, ppkGAL4, and UAS-FRT-stop-FRT-Kir2.1. In females carrying all of these transgenes, CG3542-LexA drives the expression of a flippase that removes the transcriptional stop cassette flanked by FRT sites (FRT-stop-FRT) in the UAS transgene. This means only cells expressing both CG3542-LexA and ppkGAL4 will produce Kir2.1, an inwardly-rectifying potassium channel (Johns and Marx, 1999) that suppresses neural depolarization (Fig. 3B). Indeed, although mated females with silenced CG3542-LexA and ppk-GAL4 double-positive (henceforth, CG3542-ppk) neurons lay more eggs than controls (Fig. $3 C)$, their virgin counterparts do not. The egg-laying phenotype in these females is comparable to that observed in ppk>CG3542-IR females. When we activated CG3542-ppk neurons using dTrpA1, a temperature-sensitive cation channel that depolarizes neurons at warmer temperatures $\left(30^{\circ} \mathrm{C}\right)$, we observed no change in egg-laying (Fig. 3D). It is important to note that the silencing of all ppk neurons was previously reported to induce significant egg-laying even in virgin females (See also Fig. 3E). Since, in contrast, the silencing of CG3542-ppk neurons has no effect on virgin egg-laying, we have confirmed a role for CG3542-ppk neurons in specifically regulating postmating egg-laying.

We next examined EGFP-expressing neurons in the genital tract of females carrying CG3542-LexA, LexAOP-FLP, ppkGAL4, and UAS-FRT-stop-FRT-mCD8-EGFP. Remarkably, we were only able to detect 2-3 EGFP-positive neurons per female genital tract (Fig. 3F): two in the lateral oviduct (Fig. 3F') and one in the middle oviduct near the spermatheca (Fig. 3F"). The neurons in the lateral oviduct were paired in most preparations ( $n=4 / 5)$, but the neurons in the common oviduct always appeared alone $(n=5 / 5)$. In most preparations, we were unable to detect any EGFP in the uterus $(n=4 / 5)$. To better understand the function of these CG3542-ppk neurons, we examined their polarity using the pre-synaptic marker nsyb-EGFP (Zhang et al., 2002) and the post-synaptic marker Dscam-GFP. We detected significant accumulation of nsyb-EGFP, but not Dscam-GFP in processes innervating the lateral oviduct. This indicates the CG3542-ppk neurons are likely efferent modulators of lateral oviduct contractility for controlling ovulation (Figs. 3G-3H).

Expression of a membrane-tethered SP (mSP) in either all neurons or specifically in ppk neurons suppresses mating and increases egg-laying in virgin females, recapitulating the postmating response of mated females (Nakayama et al., 1997; Rezával et al., 2012; Yang et al., 2009). Since we found the CG3542-ppk neurons regulate egg-laying, we next asked whether driving expression of $\mathrm{mSP}$ in CG3542 neurons also induces egg-laying in virgin females. To do so, we generated CG3542-GAL4 flies using the same genomic fragment we used to generate CG3542-LexA (Fig. 3A). We confirmed CG3542GAL4 driving the expression of Kir2.1 affects the egg-laying of both mated and virgin females without affecting mating receptivity (Figs. 4A-4C). This indicates CG3542-GAL4, like CG3542LexA, is expressed in neurons relevant to egg-laying. Finally, we combined CG3542-GAL4 and UAS-mSP to express mSP in the CG3542 neurons and compared their virgin egg-laying behavior with that of $p p k>m S P$ flies. We confirmed that $p p k>m S P$ virgins show elevated egg-laying compared to controls, but $C G 3542>m S P$ do not (Figs. 4D-4F). This suggests the presence of an unknown subset of ppk neurons in addition to the CG3542-ppk neurons that also regulates egg-laying. 
A

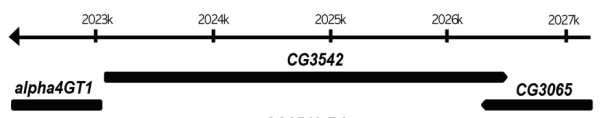

$\underset{\text { CG3542-LeXA }}{C G 3542-R A}$

B

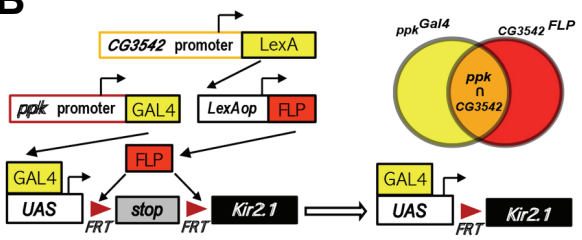

C

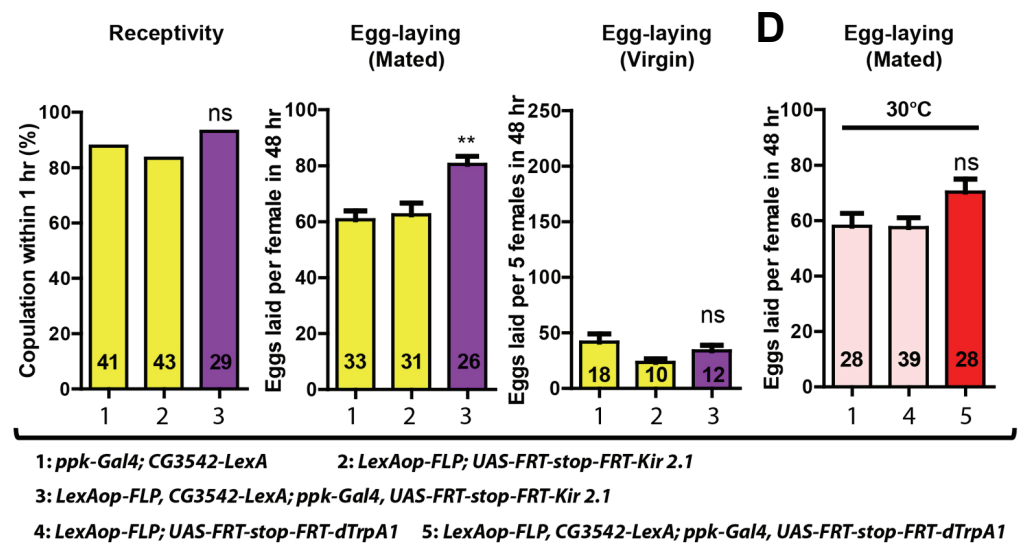

E Egg-laying (Virgin)
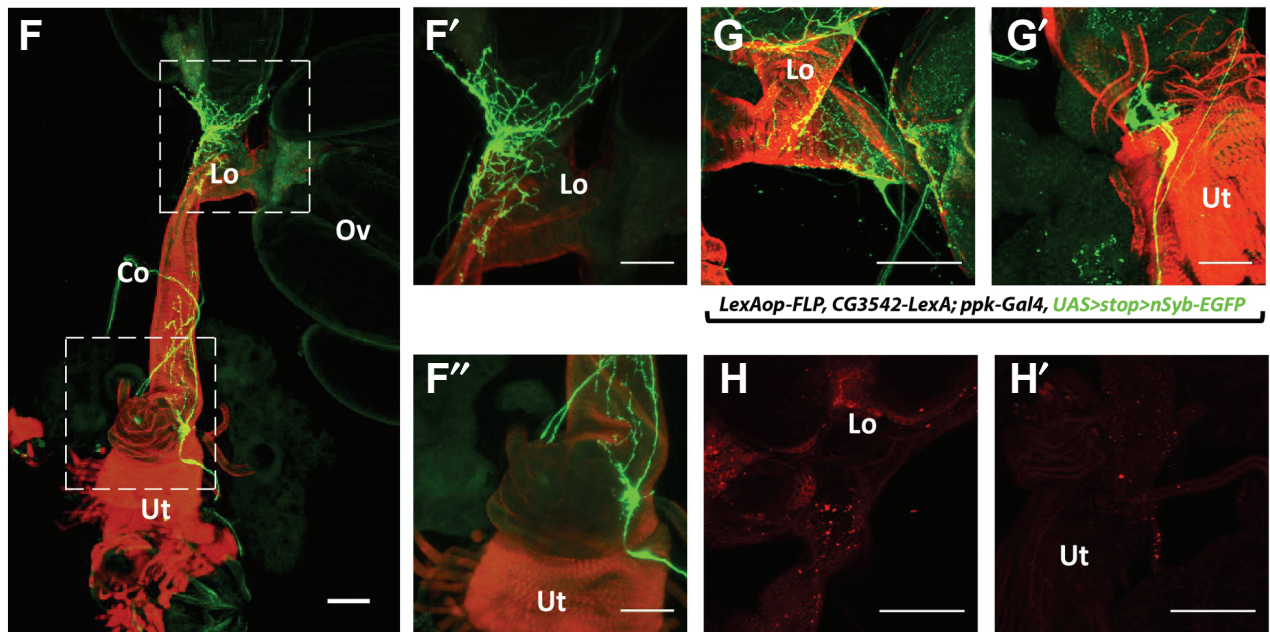

LexAop-FLP, CG3542-LexA; ppk-Gal4, UAS>stop>nSyb-EGFP
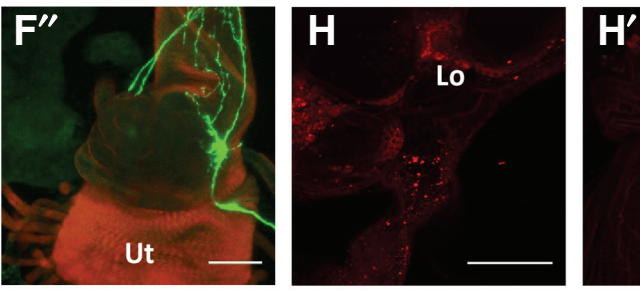

LexAop-FLP, CG3542-LexA; ppk-Gal4, UAS>stop>mCD8-EGFP

LexAop-FLP, CG3542-LexA; ppk-Gal4, UAS>stop>Dscam-GFP

Fig. 3. ppk-Gal4 and CG3542-LexA double positive neurons modulate egg-laying in mated females. (A) The CG3542 gene structure (top) and the genomic fragment used to generate CG3542-LexA (bottom). The gray and red boxes indicate untranslated and translated exons of the CG3542 transcript, respectively. (B) Schematic detailing our intersectional genetic approach. (C) Mating receptivity in virgin females (left), egglaying by mated females (middle) and egg-laying by virgin females (right) of the indicated genotypes. For egg-laying, we counted eggs laid per mated female or per five virgin females for $48 \mathrm{~h}$. The numbers inside each bar indicate $n$. Data are presented as mean percentages (for mating receptivity) or means \pm SEM (for egg-laying). ${ }^{* *} p<0.01$, not significant (ns) $p>0.05$ for comparisons against both controls (yellow bars); Fisher's exact test (mating receptivity); one-way ANOVA followed by Tukey's test for multiple comparisons (egg-laying). (D) Eggs laid per mated female of the indicated genotypes for $48 \mathrm{~h}$. The numbers inside each bar indicate $n$. Data are presented as means \pm SEM. Not significant (ns), $p>0.05$ for comparisons against both controls (pink bars); one-way ANOVA followed by Tukey's test for multiple comparisons. (E) Eggs laid per five virgin females of the indicated genotypes for $48 \mathrm{~h}$. The numbers inside each bar indicate $n$. Data are presented as means \pm SEM. ${ }^{* * *} p$ $<0.001$ for comparisons against both controls (yellow bars); one-way ANOVA followed by Tukey's test for multiple comparisons. (F) Confocal images of reproductive organs from ppk-Gal4 CG3542-LexA LexAop-FLP UAS>stop>mCD8-EGFP females, stained with an anti-GFP (green) antibody and a fluorophore-conjugated phalloidin (red). Ov, ovary; Lo, lateral oviduct; Co, common oviduct; Ut, uterus. Scale bar, $100 \mu \mathrm{m}$. $\left(\mathrm{F}^{\prime}\right.$ and $\left.F^{\prime \prime}\right)$ High magnification view of the areas outlined in $(E)$. ( $G$ and $\left.G^{\prime}\right)$ Visualization of the pre-synaptic marker nSyb-EGFP in CG3542-ppk neurons from females carrying ppk-Gal4, CG3542-LexA, LexAop-FLP, and UAS>stop>nSyb-EGFP, stained with an anti-GFP (green) antibody and a fluorophore-conjugated phalloidin (red). $(F)$, the lateral oviduct. $\left(F^{\prime}\right)$, the common oviduct. Scale bar, $100 \mu m$. $\left(H\right.$ and $\left.H^{\prime}\right)$ Visualization of the post-synaptic marker Dscam-GFP in CG3542-ppk neurons from females carrying ppk-Gal4, CG3542-LexA, LexAop-FLP, and $U A S>$ stop $>$ Dscam-GFP, stained with an anti-GFP (red) antibody and the color was presented in red. $(\mathrm{G})$, the lateral oviduct. ( $\left.\mathrm{G}^{\prime}\right)$, the common oviduct. Scale bar, $100 \mu \mathrm{m}$. 

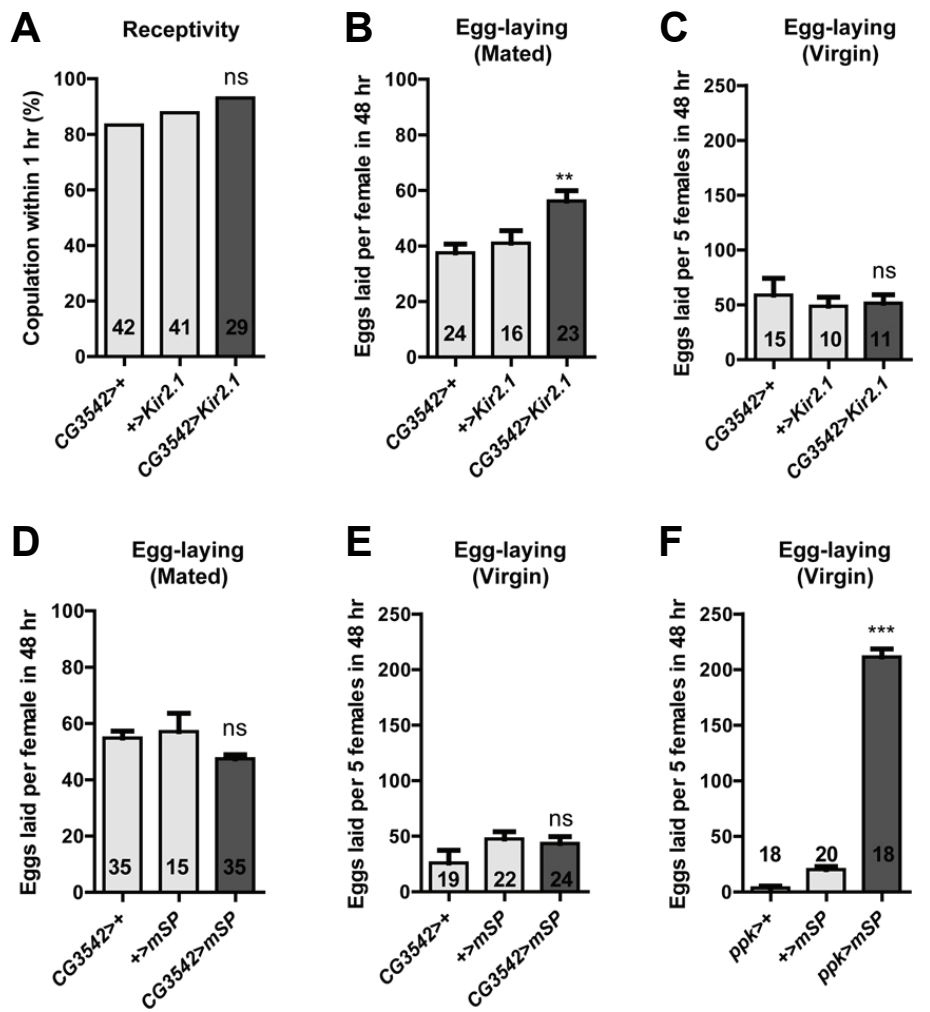

Fig. 4. SPR activation in CG3542 neurons is insufficient for inducing egg-laying in virgin females. (A) Mating receptivity of virgin females of the indicated genotypes. The numbers inside each bar indicate $n$. Data are presented as mean percentages. Not significant (ns), $p>$ 0.05 for comparisons against both controls (light gray bars); Fisher's exact test. (B, D) Eggs laid per female of the indicated genotypes for $48 \mathrm{~h}$ post-mating. The numbers inside each bar indicate $n$. Data are presented as means \pm SEM. ${ }^{* *} p<0.01$ and not significant (ns), $p>$ 0.05 for comparisons against both controls (light gray bars); one-way ANOVA followed by Tukey's test for multiple comparisons. (C, E, and F) Eggs laid per five virgin females of the indicated genotypes for $48 \mathrm{~h}$. The numbers inside each bar indicate $n$. Data are presented as means \pm SEM. ${ }^{* * *} p<0.001$ and not significant (ns), $p>$ 0.05 for comparisons against both controls (light gray bars); one-way ANOVA followed by Tukey's test for multiple comparisons.

\section{DISCUSSION}

Here, we have identified a small subset of ppk neurons that modulate egg-laying activity in female Drosophila. Using proteomic techniques we identified CG3542 as a gene whose phosphorylation is enhanced by SP treatment. We found that expression of CG3542 in ppk neurons is required to maintain normal levels of egg-laying. CG3542 encodes a protein homologous to the yeast pre-mRNA splicing factor 40 (PRP40), which associates with the $\mathrm{U} 1$ small nuclear ribonucleoprotein (snRNP) and bridges the $5^{\prime}$ and $3^{\prime}$ ends of spliced introns (Tang and Rosbash, 1996). Normal male courtship behavior was recently found to depend on the interaction between the Pelement Somatic Inhibitor (PSI) and the U1 snRNP complex. This complex affects the alternative splicing of a list of roughly 1,200 genes, including the sexually dimorphic transcription factor fruitless (fru) that regulates gender-specific behaviors (Wang et al., 2016). Although it remains unclear whether the oviduct CG3542-ppk neurons are fru-positive, one pair of lateral oviduct ppk neurons expresses fru ${ }^{\text {GAL4 }}$, a GAL4 knock-in at the fru-P1 locus (Yang et al., 2009). Thus, we speculate that the knock-down of CG3542 in the CG3542-ppk neurons alters the splicing of genes important for their development and/or function.

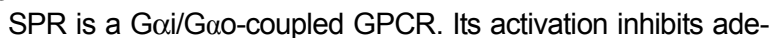
nylate cyclase, reduces intracellular cAMP, and inhibits PKA. Thus, SPR activation should produce global changes in the phosphorylation states of many proteins, particularly substrates of PKA. Indeed, our analysis identified many proteins that become hypo-phosphorylated upon SP treatment. Unexpectedly, we also identified several proteins, including CG3542, that become hyper-phosphorylated. This means SPR can promote protein phosphorylation as well as de-phosphorylation. Although the mechanism underlying this phenomenon remains unclear, it is likely SPR can affect downstream signaling pathways other than PKA. Consistent with this, over-expression in ppk neurons of an activated PKA affects mating receptivity much more strongly than it affects egg-laying (Yang et al., 2009). This suggests the distinct components of the PMR depend on distinct signaling pathways downstream of SPR. It is also of course possible that the hyper-phosphorylation of CG3542 induced by SPR activation is indirect.

Here, we report that the silencing of a small subset of ppk neurons increases post-mating egg-laying. Previously, Haesemeyer et al. (2009) found silencing all the ppk neurons has very little impact on post-mating egg-laying behavior. This discrepancy with our results may stem from a difference in the genetic reagents used to silence the ppk neurons. Haesemeyer et al. used the temperature-sensitive, dominant-negative dynamin mutant shi $^{\text {ts }}$ to conditionally block the recycling of neurotransmitter vesicles at the restrictive temperatures (Kitamoto, 2001). We, instead chose the inward-rectifying potassium channel Kir2.1 to achieve much stronger silencing.

Previous studies have suggested mating receptivity and egglaying are genetically separable in Drosophila (Yang et al., 2009). This is consistent with our observation that silencing only 2-3 CG3542-ppk neurons of the $~ 38$ ppk neurons in the female genital tract affects egg-laying but not mating receptivity. Nevertheless, we found mSP expression in the CG3542-ppk neurons is insufficient to induce egg-laying in virgin females, unlike mSP expression in all ppk neurons. Egg-laying is the culmination of a multi-step process comprising egg-formation, ovulation, fertilization, and oviposition. The anatomy of the CG3542-ppk neurons suggests they may play a role only in ovulation, because 
their pre-synaptic processes extensively innervate the basal domain of the lateral oviduct. This may explain why SPR activation in the 2-3 CG3542-ppk neurons cannot activate all steps of the process enough to increase egg-laying as a whole.

\section{ACKNOWLEDGMENTS}

We would like to thank Hae-Sook Yoon, Jungha Mun, and Ju-won Song for excellent technical assistance. We also acknowledge the Vienna Drosophila Resource Center for providing fly stocks. This work was supported by Basic Science Research Program grants to Y-J.K. (NRF-2013R1A1A2010475 and 2015R1A2A1A10054304) from the National Research Foundation of Korea (NRF), which is funded by the Republic of Korea Ministry of Science, ICT, and Future Planning (MSIP).

\section{REFERENCES}

Adams, C.M., Anderson, M.G., Motto, D.G., Price, M.P., Johnson, W.A., and Welsh, M.J. (1998). Ripped pocket and pickpocket, novel Drosophila DEG/ENaC subunits expressed in early development and in mechanosensory neurons. J. Cell Biol. 140, 143-152.

Aigaki, T., and Fleischmann, I. (1991). Ectopic expression of sex peptide reproductive behavior of female. Neuron 7, 557-563.

Beissbarth, T., Hyde, L., Smyth, G.K., Job, C., Boon, W.-M., Tan, S.-S., Scott, H.S., and Speed, T.P. (2004). Statistical modeling of sequencing errors in SAGE libraries. Bioinformatics 20 Suppl 1, i31-i39.

Bownes, M. (1989). The roles of juvenile hormone, ecdysone and the ovary in the controlof Drosophila vitellogenesis. J. Insect Physiol. 35, 409-413.

Bussell, J.J., Yapici, N., Zhang, S.X., Dickson, B.J., and Vosshall, L.B. (2014). Abdominal-B neurons control Drosophila virgin female receptivity. Curr. Biol. 24, 1584-1595.

Carvalho, G.B., Kapahi, P., Anderson, D.J., and Benzer, S. (2006). Allocrine modulation of feeding behavior by the sex peptide of Drosophila. Curr. Biol. 16, 692-696.

Chen, P.S., Stumm-Zollinger, E., Aigaki, T., Balmer, J., Bienz, M., and Böhlen, P. (1988). A male accessory gland peptide that regulates reproductive behavior of female $\mathrm{D}$. melanogaster. Cell $54,291-298$

Choi, H., Lee, S., Jun, C.-D., and Park, Z.-Y. (2011). Development of an off-line capillary column IMAC phosphopeptide enrichment method for label-free phosphorylation relative quantification. J. Chromatogr. B. Analyt. Technol. Biomed. Life Sci. 879, 29912997.

Domanitskaya, E. V, Liu, H., Chen, S., and Kubli, E. (2007). The hydroxyproline motif of male sex peptide elicits the innate immune response in Drosophila females. FEBS J. 274, 56595668.

Gruntenko, N.E., Wen, D., Karpova, E.K., Adonyeva, N. V, Liu, Y., He, Q., Faddeeva, N.V, Fomin, A.S., Li, S., and Rauschenbach, I.Y. (2010). Altered juvenile hormone metabolism, reproduction and stress response in Drosophila adults with genetic ablation of the corpus allatum cells. Insect Biochem. Mol. Biol. 40, 891-897.

Häsemeyer, M., Yapici, N., Heberlein, U., and Dickson, B.J. (2009). Sensory neurons in the Drosophila genital tract regulate female reproductive behavior. Neuron $61,511-518$

Isaac, R.E., Li, C., Leedale, A.E., and Shirras, A.D. (2010). Drosophila male sex peptide inhibits siesta sleep and promotes locomotor activity in the post-mated female. Proc. Biol. Sci. 277, 65-70.

Johns, D., and Marx, R. (1999). Inducible genetic suppression of neuronal excitability. J. Neurosci. 19, 1691-1697.

Kitamoto, T. (2001). Conditional modification of behavior in Drosophila by targeted expression of a temperature sensitive shibire allele in defined neurons. J. Neurobiol. 47, 81-92.

Liu, H., and Kubli, E. (2003). Sex-peptide is the molecular basis of the sperm effect in Drosophila melanogaster. Proc. Natl. Acad. Sci. USA 100, 9929-9933.

Monastirioti, M. (1996). Characterization of Drosophila tyramine hydroxylase isolation of mutant flies lacking octopamine maria. $\mathrm{J}$.
Neurosci. 76, 3900-3911.

Monastirioti, M. (2003). Distinct octopamine cell population residing in the CNS abdominal ganglion controls ovulation in Drosophila melanogaster. Dev. Biol. 264, 38-49.

Mount, S.M., and Salz, H.K. (2000). Pre-messenger RNA processing factors in the Drosophila genome. J. Cell Biol. 150, 37-43.

Nakayama, S., Kaiser, K., and Aigaki, T. (1997). Ectopic expression of sex-peptide in a variety of tissues in Drosophila females using the P[GAL4] enhancer-trap system. Mol. Gen. Genet. 254, 449455.

Old, W.M., Meyer-Arendt, K., Aveline-Wolf, L., Pierce, K.G., Mendoza, A., Sevinsky, J.R., Resing, K.A., and Ahn, N.G. (2005) Comparison of label-free methods for quantifying human proteins by shotgun proteomics. Mol. Cell. Proteomics 4, 1487-1502.

Park, S., Sonn, J.Y., Oh, Y., Lim, C., and Choe, J. (2014). SIFamide and SIFamide receptor defines a novel neuropeptide signaling to promote sleep in Drosophila. Mol. Cells 37, 295-301.

Peng, J., Chen, S., Büsser, S., Liu, H., Honegger, T., and Kubli, E. (2005a). Gradual release of sperm bound sex-peptide controls female postmating behavior in Drosophila. Curr. Biol. 15, 207213.

Peng, J., Zipperlen, P., and Kubli, E. (2005b). Drosophila sexpeptide stimulates female innate immune system after mating via the Toll and Imd pathways. Curr. Biol. 15, 1690-1694.

Pfeiffer, B.D., Ngo, T.-T.B., Hibbard, K.L., Murphy, C., Jenett, A., Truman, J.W., and Rubin, G.M. (2010). Refinement of tools for targeted gene expression in Drosophila. Genetics 186, 735-755.

Rezával, C., Pavlou, H.J., Dornan, A.J., Chan, Y.-B., Kravitz, E.A., and Goodwin, S.F. (2012). Neural circuitry underlying Drosophila female postmating behavioral responses. Curr. Biol. 22, 11551165.

Ribeiro, C., and Dickson, B.J. (2010). Sex peptide receptor and neuronal TOR/S6K signaling modulate nutrient balancing in Drosophila. Curr. Biol. 20, 1000-1005.

Rodri, O., Lo, I., Labarca, P., Zurita, M., Reynaud, E., and Gene, D. De (2006). Oviduct contraction in Drosophila is modulated by a neural network that is both, octopaminergic and glutamatergic. J. Cell. Physiol. 198, 183-198.

Sliter, T., Sedlak, B., Baker, F., and Schooley, D. (1987). Juvenile hormone in Drosophila melanogaster: identification and titer determination during development. Insect Biochem. 17, 161-165.

Tang, J., and Rosbash, M. (1996). Characterization of yeast U1 snRNP A protein: identification of the N-terminal RNA binding domain (RBD) binding site and evidence that the C-terminal RBD functions. RNA 2, 1058-1070.

Wang, Q., Taliaferro, J.M., Klibaite, U., Hilgers, V., Shaevitz, J.W., and Rio, D.C. (2016). The PSI-U1 snRNP interaction regulates male mating behavior in Drosophila. Proc. Natl. Acad. Sci. USA $113,5269-5274$

Walker, S.J., Corrales-Carvajal, V.M., and Ribeiro, C. (2015). Postmating circuitry modulates salt taste processing to increase reproductive output in Drosophila. Curr. Biol. 25, 2621-2630.

Yang, C.-H., Belawat, P., Hafen, E., Jan, L.Y., and Jan, Y.-N. (2008). Drosophila egg-laying site selection as a system to study simple decision-making processes. Science 319, 1679-1683.

Yang, C.-H., Rumpf, S., Xiang, Y., Gordon, M.D., Song, W., Jan, L.Y., and Jan, Y.-N. (2009). Control of the postmating behavioral switch in Drosophila females by internal sensory neurons. Neuron 61, 519-526.

Yapici, N., Kim, Y., Ribeiro, C., and Dickson, B. (2008). A receptor that mediates the post-mating switch in Drosophila reproductive behaviour. Nature 451, 33-38.

Yu, J.Y., Kanai, M.I., Demir, E., Jefferis, G.S.X.E., and Dickson, B.J. (2010). Cellular organization of the neural circuit that drives Drosophila courtship behavior. Curr. Biol. 20, 1602-1614.

Zhang, Y.Q., Rodesch, C.K., and Broadie, K. (2002). Living synaptic vesicle marker: synaptotagmin-GFP. Genesis 34, 142145.

Zhong, L., Hwang, R.Y., and Tracey, W.D. (2010). Pickpocket is a $D E G / E N a C$ protein required for mechanical nociception in Drosophila larvae. Curr. Biol. 20, 429-434.

Zhu, M.Y., Wilson, R., and Leptin, M. (2005). A screen for genes that influence fibroblast growth factor signal transduction in Drosophila. Genetics 170, 767-777. 